Background: The investigation of anti-inflammatory and immunosuppressive functions of kynurenic acid (KYNA) is now in focus. Previously, we demonstrated the opposite effects of KYNA and different KYNA analogs on tumor necrosis factor-a (TNF-a) production and tumor necrosis factor-stimulated gene-6 (TSG6) expression in U-937 monocytic cells. The potential effect of KYNA analogs on further immune mediators including alarmins (S100A12=EN-RAGE and S100A8/9=calprotectin), and on human neutrofil peptide 1-3(a-defensin) production has not been investigated.

Objectives: Therefore, in the present study, we compared the effects of newly synthesized KYNA analog on the TNF-a, alarmins and a-defensin production, correlation with the effects on the TSG-6 expression in rheumatoid arthritis (RA). Methods: 93 RA patients were involved and divided subgroups based on DAS28 activity score. Peripheral blood mononuclear cells (PBMC) was isolated from RA patients and healthy controls. As cytokine inducers heat inactivated Staphylococcus aureus (SA1) were used. In parallel in vitro experiments, the SA1 induced PBMCs were pretreated with a newly synthesized KYNA analog (compound SZR-72 was synthesized by direct amidation of KYNA). The concentrations of the above mentioned inflammatory mediators in the supernatants were quantified by using ELISA kits and the TSG-6 expression was also determined by RTqPCR method.

Results: The SA1 induced TNF-a, EN-RAGE, calprotectin and a-defensin production was significantly higher in RA patients' group than in healthy controls. KYNA analog attenuated the SA1 induced TNF-a, EN-RAGE, calprotectin and a-defensin production, and increased TSG-6 production and TSG-6 mRNA expression in PBMC cells from RA patients. The SA1 induced TNF-a and TSG-6 production correlated with the DAS28 activity score. The TNF-a inhibitory effect of the KYNA analog correlated inversely with the TSG- 6 stimulatory effect in all subgroups of RA patients based on DAS28 activity score.

Conclusion: TSG-6 expression could participate in the suppression of inflammatory cytokines, such as TNF-a, EN-RAGE, calprotectin and a-defensin. We suppose that the elevation of the TSG-6 expression by KYNA and especially by new KYNA analogs might be one of the mechanisms that are responsible for their suppressive effect on TNF-a production as a feedback mechanism in RA. KYNA and KYNA analogs have an important role in influencing TSG-6 expression, and there is a possible benefit with potential therapeutic consequence of targeting TSG- 6 expression by kynurenines in inflammatory conditions in RA. References:

[1] Mándi Y, Endrész V, Mosolygó T, Burián K, Lantos I, Fülöp F, Szatmári I, Lőrinczi B, Balog A, Vécsei L, The Opposite Effects of Kynurenic Acid and Different Kynurenic Acid Analogs on Tumor Necrosis Factor-a (TNF-a) Production and Tumor Necrosis Factor-Stimulated Gene-6 (TSG-6) Expression. Frontiers in Immunology. doi: 10.3389/fimmu.2019.01406

Acknowledgments: This work was supported by GINOP 2.3.2-2015-16-00034 Disclosure of Interests: None declared

DOI: 10.1136/annrheumdis-2020-eular.2379

\section{AB0108 \\ IRAK4 INHIBITION SUPPRESSES PROINFLAMMATORY CYTOKINE PRODUCTION FROM HUMAN MACROPHAGES STIMULATED WITH SYNOVIAL FLUID FROM RHEUMATOID ARTHRITIS PATIENTS}

A. Yadon ${ }^{1}$, D. Ruelas ${ }^{2}$, G. Min-Oo ${ }^{2}$, J. Taylor ${ }^{2}$, M. R. Warr ${ }^{2} .{ }^{1}$ Gilead Sciences, Inc., Seattle, United States of America; ${ }^{2}$ Gilead Sciences, Inc., Foster City, United States of America

Background: Rheumatoid arthritis (RA) is characterized by chronic, uncontrolled joint inflammation and tissue destruction. Macrophages are thought to be key mediators in both the initiation and perpetuation of this pathology. ${ }^{1,2}$ The RA synovium contains a complex inflammatory milieu that can stimulate macrophage-dependent production of proinflammatory cytokines through multiple signaling pathways. ${ }^{1,2}$ Existing evidence indicates that toll-like receptors (TLRs) and interleukin-1 receptors (IL-1R) along with their agonists, damage-associated molecular patterns (DAMPs) and IL-1 $\beta$, are highly expressed in RA joints and are important mediators of synovial macrophage activation and proinflammatory cytokine production. ${ }^{1-9}$ IRAK4 (interleukin-1 receptor-associated kinase 4) is a serine/threonine kinase that facilitates TLR and IL-1R signaling in many cell types, including macrophages. ${ }^{10}$ IRAK4 inhibition represents an opportunity to reduce proinflammatory cytokine production in the joints of patients with RA.

Objectives: To investigate the effect of a highly selective IRAK4 inhibitor on proinflammatory cytokine production from human macrophages stimulated with synovial fluid from patients with RA.

Methods: Primary human monocytes from 2 independent donors were differentiated for 6 days with granulocyte-macrophage colony-stimulating factor (GM-CSF) to generate human monocyte-derived macrophages (hMDMs).
hMDMs were then pretreated with an IRAK4 inhibitor for 1 hour and subsequently stimulated for 24 hours with RA synovial fluid from 5 patients. Culture supernatants were then assessed for secretion of proinflammatory cytokines by MesoScale Discovery.

Results: RA synovial fluid stimulation of hMDMs resulted in the production of several proinflammatory cytokines, including IL-6, IL-8, and TNFa. Pretreatment of hMDMs with an IRAK4 inhibitor resulted in the dose-dependent inhibition of IL-6, IL-8, and TNFa production, with an average $\mathrm{EC}_{50} \pm \mathrm{SD}$ of $27 \pm 31$, $26 \pm 41$, and $28 \pm 22 \mathrm{nM}$, respectively. Maximal percent suppression $\pm \mathrm{SD}$ of IL-6, IL-8, and TNFa were $76 \pm 8.8,73 \pm 15$, and $77 \pm 13$, respectively. To evaluate the specific IRAK4-dependent signaling pathways mediating this response, hMDMs were pretreated with inhibitors of TLR4 (TAK242) and IL-1R (IL-1RA) prior to stimulation with RA synovial fluid. Both TAK242 and IL-1RA inhibited proinflammatory cytokine production. For TAK242, maximal percent suppression \pm SD of IL- 6 , IL-8, and TNFa were $39 \pm 25,48 \pm 24$, and $50 \pm$ 21 , respectively. For IL-1RA maximal percent suppression \pm SD of IL-6, IL-8 and TNFa were $18 \pm 18,20 \pm 23$, and $16 \pm 18$, respectively. The broad range of inhibition across each stimulation highlights the complexity and variability in the signaling pathways mediating proinflammatory cytokine production from hMDMs stimulated with RA synovial fluid, but demonstrates that RA synovia fluid can stimulate proinflammatory cytokine production in hMDMs, at least partly, through IRAK4-dependent pathways.

Conclusion: This work demonstrates that IRAK4 inhibition can suppress proinflammatory cytokine production from macrophages stimulated with synovial fluid from patients with RA and supports a potential pathophysiological role for IRAK4 in perpetuating chronic inflammation in RA.

\section{References:}

[1] Smolen JS, et al. Nat Rev Dis Primers. 2018;4:18001.

[2] Udalova IA, et al. Nat Rev Rheumatol. 2016;12(8):472-485.

[3] Joosten LAB, et al. Nat Rev Rheumatol. 2016;12(6):344-357.

[4] Huang QQ, Pope RM. Curr Rheumatol Rep. 2009;11(5):357-364.

[5] Roh JS, Sohn DH. Immune Netw. 2018;18(4):e27.

[6] Sacre SM, et al. Am J Pathol. 2007;170(2):518-525.

[7] Ultaigh SNA, et al. Arthritis Res Ther. 2011;13(1):R33

[8] Bottini N, Firestein GS. Nat Rev Rheumatol. 2013;9(1):24-33.

[9] Firestein GS, Mclnnes IB. Immunity. 2017;46(2):183-196.

[10] Janssens S, Beyaert R. Mol Cell. 2003;11(2):293-302.

Disclosure of Interests: Adam Yadon Employee of: Gilead, Debbie Ruelas Employee of: Gilead, Gundula Min-Oo Employee of: Gilead, James Taylor Employee of: Gilead, Matthew R. Warr Employee of: Gilead DOI: 10.1136/annrheumdis-2020-eular.3793

\section{AB0109 THE ROLE OF CD70 IN THE DEVELOPMENT OF RHEUMATOID ARTHRITIS}

S. J. Yoo ${ }^{1}$, S. W. Kang ${ }^{1}$, J. Kim ${ }^{1}$, I. S. Yoo ${ }^{1}$, C. K. Park ${ }^{1}$, H. R. Lee ${ }^{1} .{ }^{1}$ Chungnam National University Hospital, Daejeon, Korea, Rep. of (South Korea)

Background: Rheumatoid arthritis (RA) is a progressive, chronic inflammatory autoimmune disease. Pro-inflammatory molecules, activated lymphocytes, and the migration of inflammatory cells are important in the development of RA There are many unknown causes of RA. And there are many patients who are refractory to treatment with known disease-modifying anti-rheumatic drugs. So, unknown cause of RA needs to be elucidated.

CD70 is a member of the tumor necrosis factor (TNF) superfamily and a ligand for CD27. The interaction of CD70 with its receptor CD27 promotes expansion and differentiation of memory and effector $\mathrm{T}$ cells as well as B-cell expansion and plasma cell differentiation. Hypoxia is an important micro-environmental factor in RA synovium. Hypoxia induces activation of hypoxia inducible factor (HIF). The expression of HIF-2a is up-regulated in human RA synovium. Reactive oxygen species (ROS) has been implicated in the pathophysiology of RA.

Objectives: In this study, we tried to examine the presence of CD70 in RA synovium and investigate the role of CD70 in the development of RA associated with HIF-2 $a$ and ROS.

Methods: Fibroblast-like synoviocyte (FLS), peripheral blood (PB) and synovial fluid (SF) were used for experiments. FLS was stimulated with recombinan human (rh)-IL-17 and rh-TNF-a. N-acetyl-L-cysteine (NAC) was used as a ROS scavenger. HIF-2a inhibitor (PT-2385) was used for examine the effect of HIF-2a in RA-FLS. RT-PCR, qPCR, western blotting, flow-cytometry, ELISA, cell migration assay, and scratch wound assay were performed

Results: CD70 mRNA is present and elevated by stimulation with IL-17 and TNF-a in both RA-FLS and osteoarthritis (OA)-FLS (Fig 1). CD70 also expresses on the surface of RA-FLS and OA FLS (Fig 2). CD70 expression on the surface of FLS is elevated by stimulation with IL-17 and TNF-a in both 
RA and OA. Soluble CD27 is present higher in the supernatant of RA-SF than OA-SF (Fig 3). HIF-2a mRNA, HIF-2a protein, and the amount of ROS were all elevated after treatment with IL-17 and TNF-a in RA-FLS (Fig 4, Fig 5). CD70 expression and the amount of ROS were lowered by treatment with HIF-2a inhibitor in RA-FLS (Fig 6). Decreased amount of ROS results in decreased CD70 expression on the RA-FLS (Fig 7). CD70 influenced on cell migration directly or by HIF-2a (Fig 8).
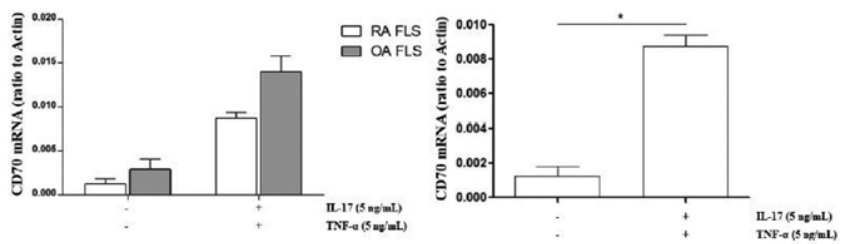

Figure 1. CD70 mRNA in RA-FLS and OA-FLS

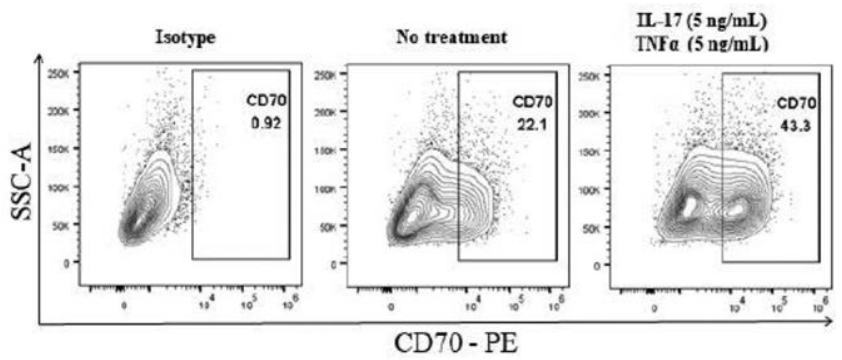

Figure 2. CD70 expression on the surface of RA-FLS

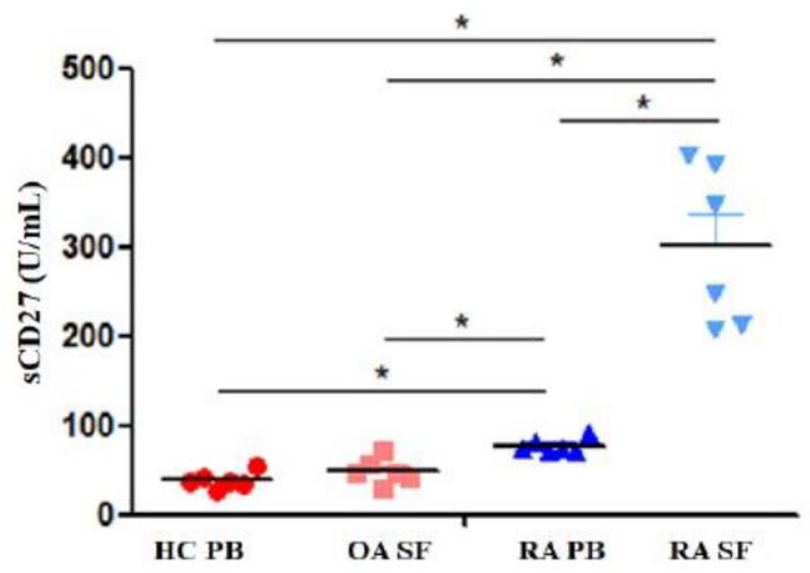

Figure 3. The amount of sCD27 by ELISA

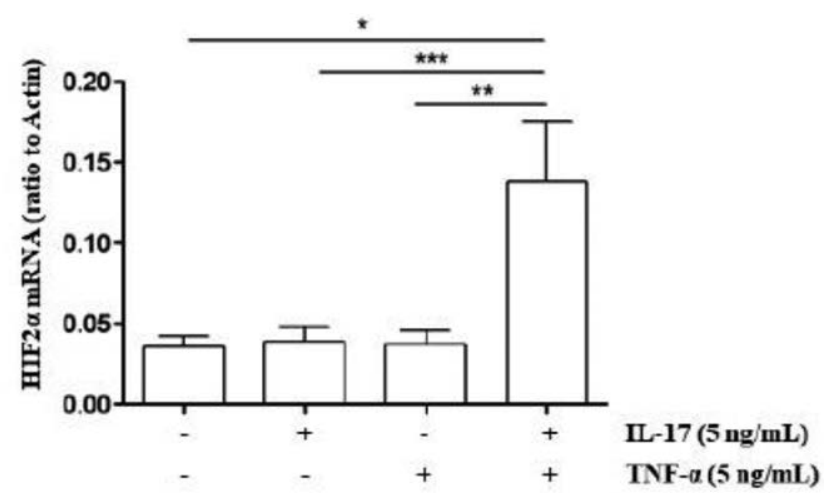

Figure 4. HIF-2 $\alpha$ mRNA in RA-FLS

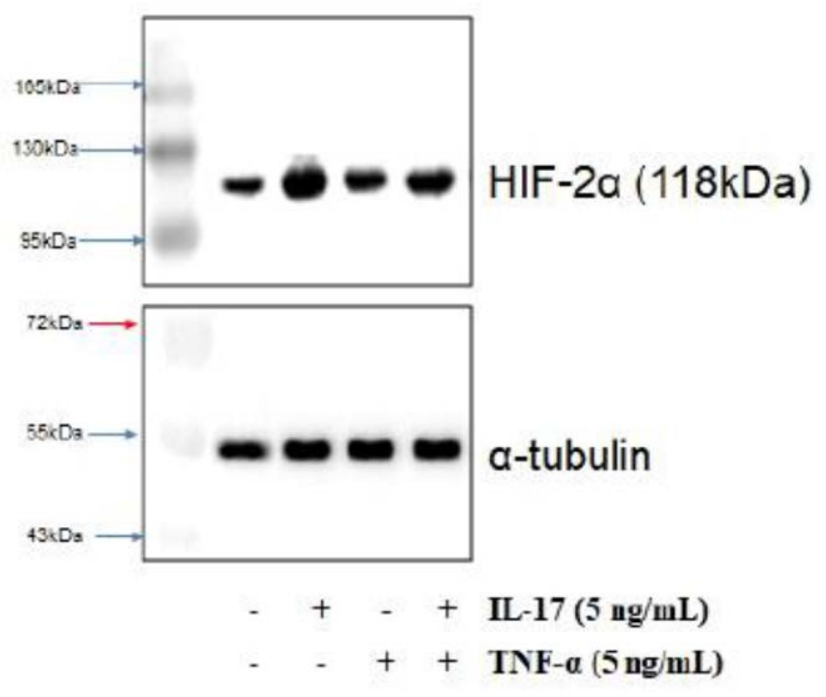

Figure 5. HIF-2a protein in RA-FLS

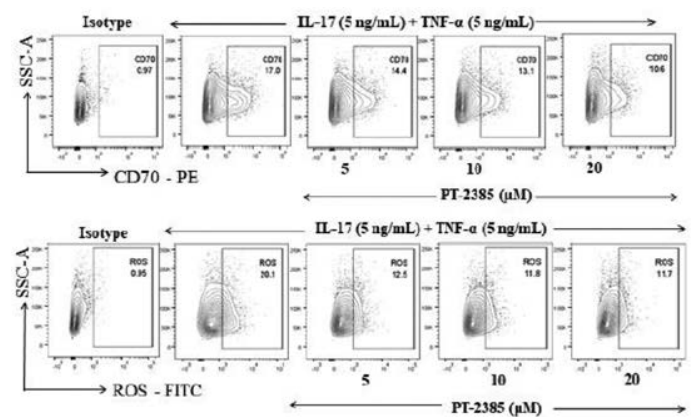

Figure 6. CD70 expression and ROS on the surface of RA-FLS by treatment with HIF-2 $\alpha$ inhibitor. 


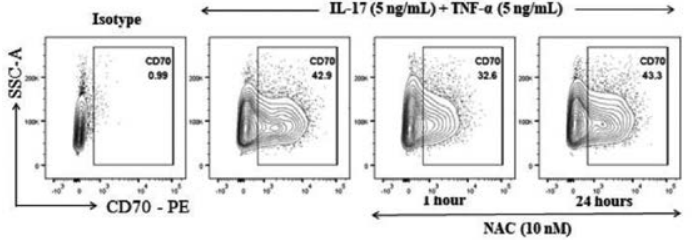

Figure 7. CD70 expression on the surface of RA-FLS by treatment with ROS scavenger
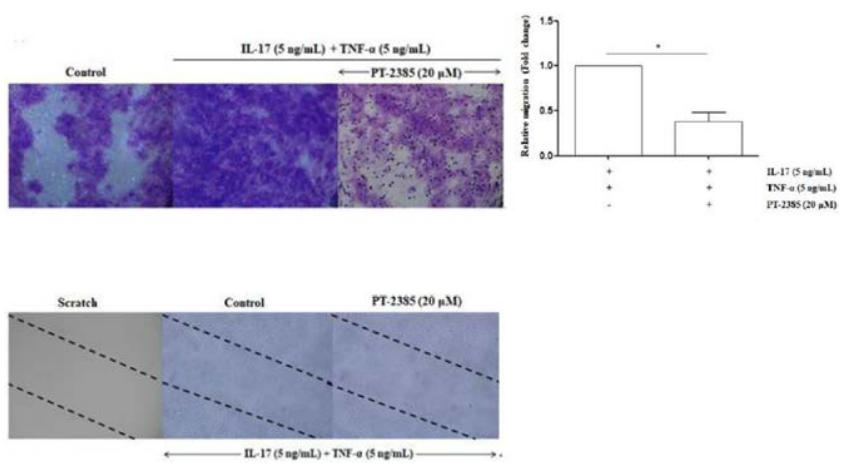

Figure 8. Cell migration in RA-FLS by treatment with HIF-2 $\alpha$ inhibitor

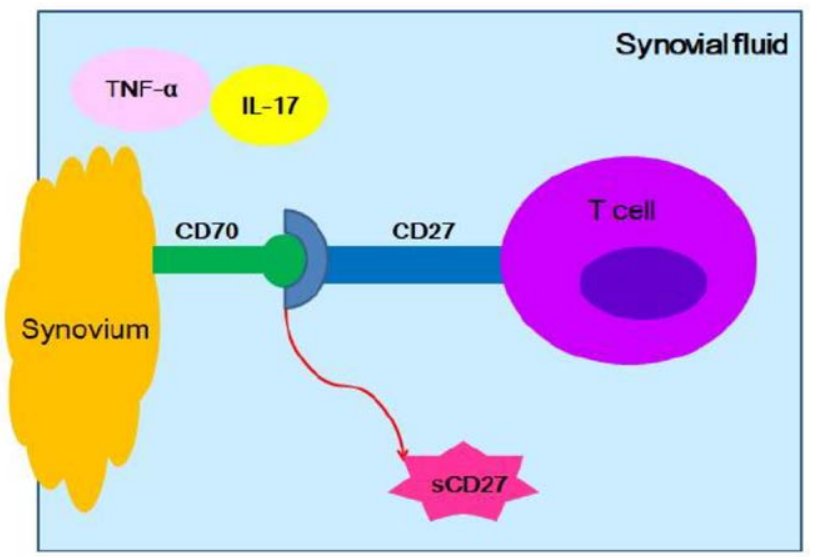

Figure 9. CD70-CD27 interaction on RA-FLS

Conclusion: In this study, we found the function of CD70 in RA-FLS associated with HIF-2 $a$ and ROS. First, CD70 on RA-FLS interacts with CD27 in the RA-SF and this interaction produces SCD27 (Fig. 9) and CD70 has an influence on the migration of RA-FLS. Second, IL-17 and TNF- $\alpha$ are critical factors to trigger the expression of CD70, HIF-2a and ROS in RA synovium. Third, CD70 is regulated by HIF-2a associated with ROS. From these results, we suggest that CD70 may be a new therapeutic target of RA. And SCD27 also may be an important diagnostic maker of RA.

References:

[1] Lundy SK, Sarkar S, Tesmer LA, Fox DA. Cells of the synovium in rheumatoid arthritis. T lymphocytes. Arthritis Res Ther. 2007;9(1):202.

[2] Nevius E, Gomes AC, Pereira JP. Inflammatory Cell Migration in Rheumatoid Arthritis: A Comprehensive Review. Clin Rev Allergy Immunol. 2016;51(1):59-78.

[3] Bowman MR, Crimmins MA, Yetz-Aldape J, Kriz R, Kelleher K, Herrmann S. The cloning of CD70 and its identification as the ligand for CD27. J Immunol. 1994;152(4):1756-61.

[4] Kitajima S, Lee KL, Fujioka M, Sun W, You J, Chia GS, et al. Hypoxia-inducible factor-2 alpha up-regulates CD70 under hypoxia and enhances anchorage-independent growth and aggressiveness in cancer cells. Oncotarget. 2018;9(27):19123-35.
[5] Gaber T, Dziurla R, Tripmacher R, Burmester GR, Buttgereit F. Hypoxia inducible factor (HIF) in rheumatology: low O2! See what HIF can do! Ann Rheum Dis. 2005;64(7):971-80.

Disclosure of Interests: None declared

DOI: 10.1136/annrheumdis-2020-eular.2448

\begin{tabular}{|l|l}
\hline AB0110 & ADIPONECTIN INDUCES PRO-INFLAMMATORY \\
CHEMOKINE AND CYTOKINE PRODUCTION BY \\
PERIPHERAL BLOOD MONONUCLEAR CELLS AND \\
FIBROBLAST-LIKE SYNOVIOCYTES FROM NON- \\
INFLAMED SUBJECTS
\end{tabular}

Y. Zhang ${ }^{1}$, J. Aldridge ${ }^{1}$, G. K. Vasileiadis ${ }^{1}$, A. C. Lundell ${ }^{1}$, A. Rudin ${ }^{1}$, C. Maglio' ${ }^{1}$. ${ }^{1}$ University of Gothenburg, Dep. of Rheumatology and Inflammation Research, Gothenburg, Sweden

Background: Adiponectin is a cytokine mainly secreted by the adipose tissue ${ }^{1}$, whose circulating levels are paradoxically low in subjects with obesity and associate with a beneficial metabolic profile ${ }^{2}$., Recent studies have shown that adiponectin levels are elevated in both serum and synovial fluid collected from patients with rheumatoid arthritis $(\mathrm{RA})^{3,4}$. Moreover, adiponectin is able to induce the production of interleukin (IL)-6, tumor necrosis factor (TNF), CXCL1 and CXCL8 by lymphocytes from healthy subjects ${ }^{5}$, and of IL-6 and CXCL8 by fibroblast-like synoviocytes (FLS) from patients with RA ${ }^{6}$. However, it is not clear if adiponectin is able to initiate the inflammatory processes associated with the preclinical phase of RA.

Objectives: We aim to determine if adiponectin is able to induce inflammatory responses in peripheral blood mononuclear cells (PBMCs) and FLS from non-inflamed subjects.

Methods: Human PBMCs were collected from healthy donors, whereas non-inflamed FLS from non-arthritic patients who underwent diagnostic arthroscopy due to previous trauma. PBMCs $\left(1 \times 10^{5}\right.$ cells/well in 96-well plate) and FLS (5000 cells/well in 96-well plate) were stimulated using $5 \mu \mathrm{g} / \mathrm{ml}$ recombinant human total adiponectin protein, and the supernatants were collected 48 hours after stimulation. Phytohemagglutinin (PHA) and TNF were used as positive controls to activate PBMCs and FLS, respectively. Using multiplex assay and ELISA, we screened the production of 13 chemokines and 12 cytokines from healthy human PBMCs and non-inflamed FLS.

Results: Adiponectin was able to stimulate a distinct profile of chemokines and cytokines in PBMCs and FLS. In both healthy PBMCs and non-inflamed FLS adiponectin induced the production of CXCL1, CXCL5, CXCL8, CCL2 and IL-6. Moreover, CCL3, CCL20, CCL4, CCL17, TNF, IL-10 and GM-CSF were induced by adiponectin only in healthy PBMCs, whereas CXCL10, CCL5 and CCL11 only in non-inflamed FLS (Fig. 1 and 2).
A

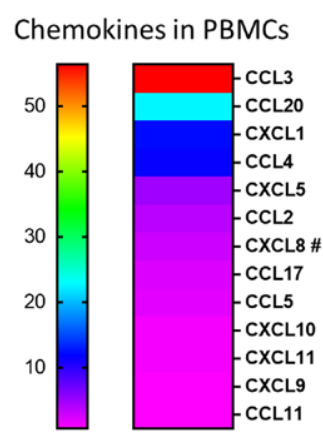

Mean fold change adiponectin vs unstimulated control
Chemokines in FLS

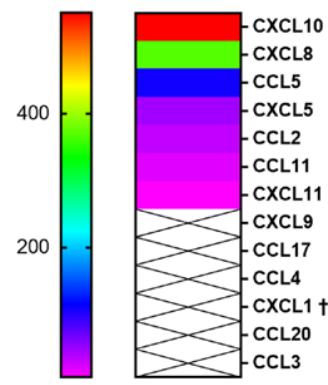

Mean fold change adiponectin vs unstimulated control
Figure 1. Adiponectin induces the production of various chemokines from PBMCs $(A)$ and FLS (B). CXCL8 (\#) was measured using ELISA, and other chemokines were measured using multiplex assay. The fold change of CXCL1 in FLS ( $\dagger$ ) was not calculated because its level before stimulation was undetectable.

Conclusion: We here report that adiponectin has pro-inflammatory properties as it induced chemokine and cytokine production from human healthy PBMCs and non-inflamed FLS. As adiponectin is able to induce pro-inflammatory responses from non-inflamed cells, we suggest that this adipokine might be implicated in the preclinical phase of RA pathogenesis.

References:

[1] Makki K, Froguel P, Wolowczuk I. Adipose tissue in obesity-related inflammation and insulin resistance: cells, cytokines, and chemokines. ISRN Inflamm 2013;2013:139239. 